\title{
A Review: Electric Field Sensing for Human-Computer Interaction Applications
}

\author{
Atika Arshad ${ }^{1}$, Ahmad Fadzil Ismail ${ }^{2}$, Sheroz Khan ${ }^{3}$, Wahidah Hashim ${ }^{4}$ and \\ Mohammad Kamrul Hasan ${ }^{5}$ \\ 1, 2, 3, 5 Department of Electrical and Computer Engineering, International Islamic \\ University Malaysia, Malaysia \\ ${ }^{4}$ Department of System \& Networking, Computer Science \& Information \\ Technology, Universiti Tenaga Nasional, Malaysia \\ latikaarshad@hotmail.com
}

\begin{abstract}
Human- computer interaction (HCI) in invasive computing system has been proving as a dynamic research interest since the late nineties. The interference of a human body intercepting the way between a transmitting electrode having low frequency and a receiving electrode changes the displaced current which is computed at the receiving electrode. The procedure of human body interfacing with electric fields of low frequency could be implemented to make an extensive area of interactive fields namely human computer interface, virtual reality, interactive surfaces as well as automotive applications. This paper aims to make an overview of some electric field sensing techniques along with their applications including Human-Computer Interaction (HCI) procedure as well as the evaluation of the robustness of the applied techniques.
\end{abstract}

Keywords: Electric field, Capacitive sensor, Human computer interaction and Passive devices

\section{Introduction}

Technology is an on-going development and one of its essential and critical goal is to increase the ease and flexibility of any system. The demand for enhancing techniques has directed researchers to explore and expand the use of computers in control systems.

Human-Computer Interaction (HCI) has become one of the most significant ongoing research which has been rapidly growing over the last two decades. HCI is a system which uses Electric Field Sensing (EFS) that involves the interaction between the computer and the user. The electrostatic behavior of a human body is operated with EFS. Generally, it communicates directly with the human body and its properties as physical matter such as capacitance and conductance. EFS calculates capacitance to sense the closeness of the human body. Capacitance forms the charge stored between transmitting and receiving electrodes. This work aims to focus on the merits and demerits of capacitive sensing techniques for human gesture identification. Moreover, this paper gives an overview of the existing approaches in electric field sensing for Human- computer interaction (HCI). Research carried out in the area of electrical field sensing have become a renewed interest among researchers currently for touch location detection, activity recognition, and gesture identification.

\section{Overview on Electric Field Sensing}

Electric field between two objects can be modeled with a capacitor, hence capacitor is responsible for storing energy within an electric field. The simplest way to model a capacitor is by placing two parallel conductors distanced by a space with an insulating 
dielectric material. Any conductive materials can be used as the plates of a capacitor which makes it very unobtrusive and simply integrated on the floor. The electric field of a capacitor may vary based on the material located between two plates, which may "dampen" the field. The damping can be varied based on the molecular properties of the material and its structure and shape. It can be quite easy like the linear dependence in the parallel plate capacitor, but also arbitrarily complex relationship can reflect upon detailed shapes (with cavities) and inhomogeneities in the molecular properties. The main equation for active capacitive sensing is denoted as $C=Q / V$ where $\mathrm{Q}$ (stored charge) and Voltage $\mathrm{V}$ can be easily computed, and $\mathrm{C}$ varies on the properties of an object including what is hidden inside it. Thus, an object of varied structure, cavities, or different surface will vary Capacitance C. Therefore, we can "look" inside an object in a non-invasive way by computing two electric parameters. Apparently, the information that we receive is not unlimited: a single scalar value. As a result, the computation of capacitance due to a single electrode pair cannot necessarily explain complicated structural information. Nevertheless, it can signify structural variation within the objects.

The relation between different properties of the capacitor can be shown as follows:

$$
\begin{aligned}
E & =V / d \\
E & =Q / \varepsilon A
\end{aligned}
$$

where $\mathrm{E}$ is denoted as electric field strength, $\mathrm{V}$ the voltage exists between the conductive plates, $\mathrm{d}$ the distance between the two plates, $\mathrm{Q}$ is the charge of the capacitor, $\varepsilon$ the dielectric permittivity of the material between the conductive plates and A the surface area of the plates. The formula can further be derived to

$$
V=d Q / \varepsilon A
$$

which indicates that the change in voltage is directly proportional to its distance and the inversely proportional to its area, assuming that the charge of the object and the permittivity remain constant. Similarly, it can also be assumed that the voltage between environmental objects and the ground is constant, in order to sense the movement of the body by equating the voltage of the body with respect to ground.

\section{Modes of Electric Field Sensing}

The knowledge of applying electric fields to localize humans was first introduced by Zimmerman and his team [1], in the year 1995. He proposed two modes of operation for electric field localization including the human shunt and the human transmitter. In 1998, Smith, et al. [2] presented a third operating mode: the loading mode. Figure 1 illustrates a lumped circuit model [3] which describes all three modes of field sensing for a single transmitting-receiving pair with a single object. These methods use different combinations of emitting and sensing electrodes to determine distance, movement, and orientation of a human body. 


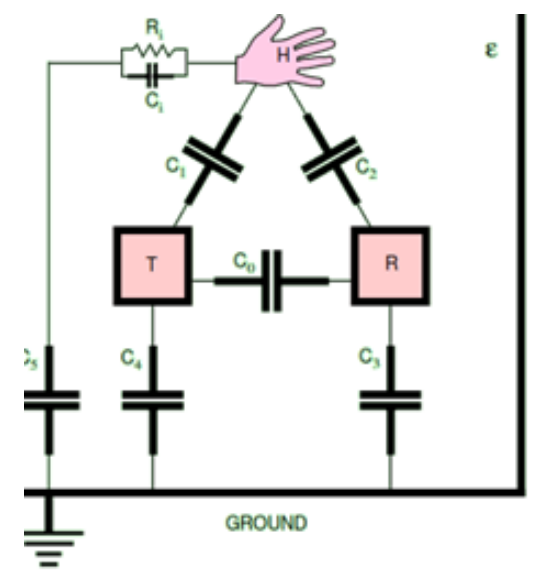

Figure 1. Lumped Circuit Model of Electric Field Sensing Parameters [3]

The model is made up of two electrodes; a transmitter (T) driven by a low voltage, low frequency $\sim 50 \mathrm{~Hz}$ signal, a receiver (R) that identifies the transmitted signal through the capacitive paths, and a field-altering object: a human hand.

1) Loading Mode: As the object being sensed approaches or moves away from the transmitter, the value of capacitance $\mathrm{C} 1$ changes significantly, thus, the movement can be sensed by measuring the increase / decrease of the displacement current. Further, by sensing the level of this variation, proximity or closeness can be detected. Loading mode is a modification of the method used in Theremin. The advantages of this mode are that only a single electrode is required (no receiver is required) [3] to measure the current displacement between the transmitting electrode and the object, thus the burden of installation is minimized, also the electrodes could be simply shielded against the effect of current electric potentials in the neighbourhood. Loading mode sensors are particularly appropriate to implement bigger sensing systems for activity recognition or entire body interaction.

2) Transmit Mode: Here, the object acts like the transmitter itself, or is capacitively linked to the transmitter through $\mathrm{C} 1$. The movement of Object has an impact on the effective distance between transmitter and receiver, and this creates measurable changes in $\mathrm{C} 2$ [3]. The positive aspect of this mode pro the transmitted signal to be picked up by one or more receivers. The measured signal is considered to be dependent on the distance exists between the receiver ground electrode and the person. The disadvantage of this mode involves the user to be connected to an electrode hence multiple electrodes are required to be installed in an area for detection.

3) Shunt Mode: In shunt mode, there is no connection between object and transmitter or receiver. Without the presence of an object, a displacement current flows through $\mathrm{C} 0$. When an object is present in the field, the values of $\mathrm{C} 0, \mathrm{C} 1$ and $\mathrm{C} 2$ vary. This leads to some changes in the whole displacement current passing from transmitter to receiver [3].

Through using multiple electrodes and multiplexing the transmitter, a number of measurements can be conducted. From these measurements and assumptions, information related to the location and orientation of object inside the field can be assumed.

Among the three modes of electric field sensing, shunt mode provides maximum information. In the case of transmitting and loading modes, $\mathrm{n}$ measurements can be conducted using $n$ amount of transceivers, while in shunt mode $n(n-1) / 2$ measurements can be conducted with n number of electrodes. Every measurement provides distinctive information related to the geometry of the situation [2]. The limitation of using shunt mode is that the coupling between the object and the ground has an effect on the 
measurement outputs, therefore it's difficult to accurately measure the distance from the body to the electrode. Henceforth, [3] this method is not able to differentiate the difference between a small object close by and a big object far away. Nonetheless, having a numerous number of transmitting or receiving electrodes will halt the degeneracy.

\section{Related Work}

Human-Computer Interaction (HCI) can be fundamentally defined as the research field that investigates the link between humans and their technological environment. In particular, the approach studied for HCI is basically the study on the influence of generation, reception and distortion of electric fields by the human body. Elephantnose Fish (Gnathonemus petersii), a weakly electric fish can self generate weak electric fields [4-5] to correctly determine the spatial location, conductivity, object size, shape, distance, and velocity of nearby objects for social communication, recognizing sex, age, and dominance hierarchy. The fields are created by an electric organ in the tail and are sensed by an electroreceptive surface on big parts of the body.

The static electric fields from charged objects cannot make any sensible motion tracking techniques. This happens because of the complications of maintaining the charges on the object from leaking off into air, and the probability that other objects can inadvertently have a static charge. Nevertheless, by making use of oscillating electric fields at extremely low frequencies, these issues are solved. The simple capacitance measuring electronic circuit can sense the distance between conductive electrodes.

Theremin [6] was first to introduce an artificial electric field sensing system for a musical instrument that played without touch. The user's capacitance is measured by the instrument with relative to a set of a transmitting and receiving antennae. Two set of antennas are used; one to control the amplitude (volume) and other for the frequency / pitch of the sound produced. Hence, The Theremin is the primary device for determining the position of an object (player's body parts) by calculating the variations in an emitted electromagnetic field.

Regardless the amount of research undertaken over the years on capacitive sensing, only a very little research work has been reported on positioning and tracking humans via capacitive sensing technique. Karlsson \& Järrhed [7] were the first to develop a simple electrode configuration using transmit mode designed to identifying movement and detect the presence of a person the presence or movement of a person approaching nearby a robot. The system was designed to halt the machine in case a moving person comes too close to it, in order to ensure safety. Following the "person detector", a person sensing room was modeled [3] which was also based on transmit mode. Here a transmitter electrode covers the floor of the Person Sensing Room and four-receiver electrodes were positioned on the walls. By using the floor electrode, a low-frequency signal was given to a person, and the signal strength originating from the person was computed with the wall electrodes. The room was capable of identifying the position of a person on the floor by using the measured signal strength.

The DiamondTouch system [8] generates various signals at various sections of a table and differentiates the touch position by indicating the corresponding signal received by the user. DiamondTouch employs the transmitting mode electric field sensing. The design of DiamondTouch is accomplished by placing a grid of metal strips (transmitters) underneath a table and different sets of the grid (receivers) positioned in the chair of each user. As a user touches the table, the strips of the grid connected to the table emanate an electric field which completes a capacitively coupled circuit. The receiver can then identify the specific part of the table surface the user is in touch with. Nevertheless, the DiamondTouch table is only able to sense when the user makes a direct skin contact to the table. 
In, [9] a novel, robust, low cost and contactless gesture recognition system named Thracker using capacitive sensing was reported by Wimmer. The system was considered to be of low cost as only standard electronic components like a few typical ICs were used and the usual USB interface chip accessible. The system proposed by Thracker is able to detect grasp gestures and recognize hand position of the users.

Wimmer et al. [10] further researched on capacitive sensing technology for prototyping human-computer interaction. He developed a CapToolKit, implementing loading mode for detecting the presence of humans, objects and also for integrating three-dimensional interaction with everyday objects. He built a table (CapTable) with capacitive sensors ( 8 metal plates) mounted below the table to sense activities such as the number of people seated at the table, the sitting configuration, activity of a person; whether a person is approaching the table or simply passing by it.

Valtonen et al. [11-12] demonstrated the TileTrack system, the system is able to track more than one person by determining the capacitance amongst the numerous floor tiles. Transmit mode of electric field sensing was implemented, His system measured the capacitance between the electrodes by determining the current flow at the receiver electrode at an instant when a person is positioned on a tile. Figure2 and Figure3 demonstrates the capacitance model of the system and capacitance measurement model of the system respectively. The Tile Track system operates as; $\mathrm{C}_{\mathrm{o}}$ denotes the capacitance offset (constant) in the absence of the person. $C_{F}$ represents the foot. $C_{R}$ indicates the receiver capacitance formed between the human feet and the receiver electrode. If someone goes inside the tracking area, $\mathrm{C}_{\mathrm{F}}$ and $\mathrm{C}_{\mathrm{H}}$ vary. If a person stands on a tile, the value of $\mathrm{C}_{\mathrm{E}}$ rises and the transmitted signal becomes coupled to the person's body. After that, this signal passes through $\mathrm{C}_{\mathrm{H}}$ to the receiving electrode, and the system measures this current flow. The system measures the series capacitance of $C_{E}$ and $C_{R}$ parallel to $C_{o}$, the total capacitance from the measured current. The system incessantly scrutinizes the tiles one at a time and conducts the measurement of whole capacitance between the tile in question and the receiving electrode. It indicates the user's position by making a comparison of the measured values to the calibration values. This procedure makes use of a $32 \mathrm{kHz}$ measurement frequency, at which the human body operates as an appropriate conductor.

A precision of $15 \mathrm{~cm}$ was obtained for a standing position, and $42 \mathrm{~cm}$ accuracy for a person when walking. Furthermore, this system is able to operate even when the tiles are covered with insulating material, like carpets or when the user is wearing shoes. However, two issues are detected namely accuracy and many people detection. Confusion occurs if another person comes in between the test human and receiver since the electric-field will escape using another person as they go by. Due to these problems, this system is inappropriate for human location approximation in day to day life.

In, [13] a passive system was proposed based on transmit mode low frequency capacitive coupling. The system presented measures the height and posture of a user. The electrodes are placed such that transmitting electrode is on the floor surface and the receiver electrode is horizontally on the ceiling. With this mode of setup, the capacitance measured between the human body and ceiling electrode is inversely proportion to the distance between them. The height of the person can be found by converting the capacitance to an absolute distance using capacitance-to-height conversion function. Furthermore, the posture of a person can also be obtained by calculating the percentual height of the person from the standing height with equation provided by the author, hence the actual posture of a person is determined by comparing the results with a predefined classification of the posture. Valtonen's system confirmed to be able to measure a person's height at an accuracy of $90 \%$ with $5.2 \mathrm{~cm}$ precision when a person is standing and with $14.3 \mathrm{~cm}$ precision in other postures. 


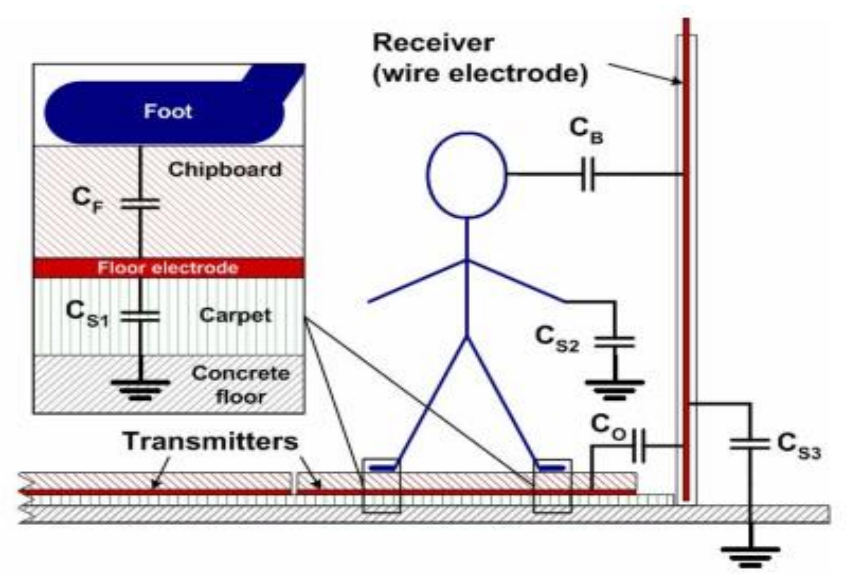

Figure 2. Capacitance Model of the System [13]

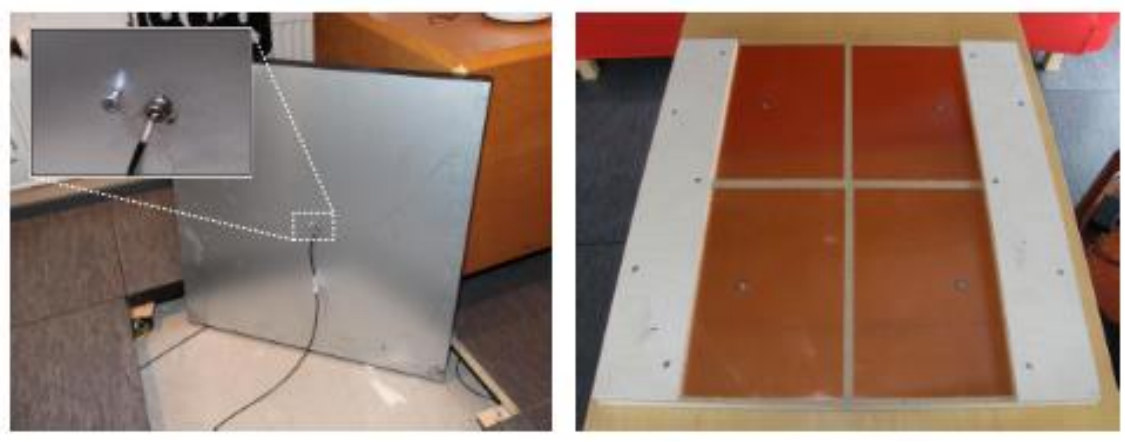

Figure 3. Capacitance Measurement Model of the System [13]

The Electric Sensors with Intelligence (ELSI) system [14], operates in loading mode. It tracks people by using the near field imaging (NFI) technology. It conducts the measurement of capacitance that exists between a transmitting electrode and the ground [13]. The computed signal is given to a transmitter at once keeping the other sensors grounded. As soon as a person comes near the actuated electrode, the displacement current flowing from the object to the adjoining grounded sensors is enhanced. The system measures the displacement current from each sensor making use of the current and the location of the sensors for determining the person's position.

The Electric Sensors with Intelligence (ELSI) system is based on the use of near field imaging (NFI) to track people which operate in the loading mode; it conducts the measurement of capacitance that exists between a transmitter and the ground [14]. The measured signal is given to a transmitter at once; when the other sensors are grounded. When a conductive object is present (a person) near to the actuated electrode, the displacement current passing from the object towards the adjacent grounded sensors is increased. The system carries out the measurement of the displacement current from every single sensor, makes use of the current and the physical location of the sensors to identify the person's position. Figure 4 illustrates the ELSI measurement basics.

The electrodes are $30 \times 30 \mathrm{~cm}$ in size and metal thick-film squares those of which are mounted underneath the floor. As a result, the system is fully untraceable. The covered floor area size is scalable. However, the pilot platform explained in [14] was $4.5 \times 4.0 \mathrm{~m}$ in size. This pilot attained a mean location error of $21 \mathrm{~cm}$ for five persons in walking state. Moreover, the advancement of the ELSI system has created systems that are appropriate for health care applications and are capable of identifying when a person falls suddenly [15]. Nowadays ELSI systems are commercially obtainable and are being used 
in applications like nursing homes. Installation and construction expenses for ELSI systems are almost same as TileTrack systems. The electronics and electrodes are quite cost effective. However, the installation is complicated and costly, particularly if mounted on an existing home. The disadvantage of this system can be said as its' incapability to recognize the person or object which has been positioned. Because the system measures only electrical parameters from the human body, it cannot identify people.
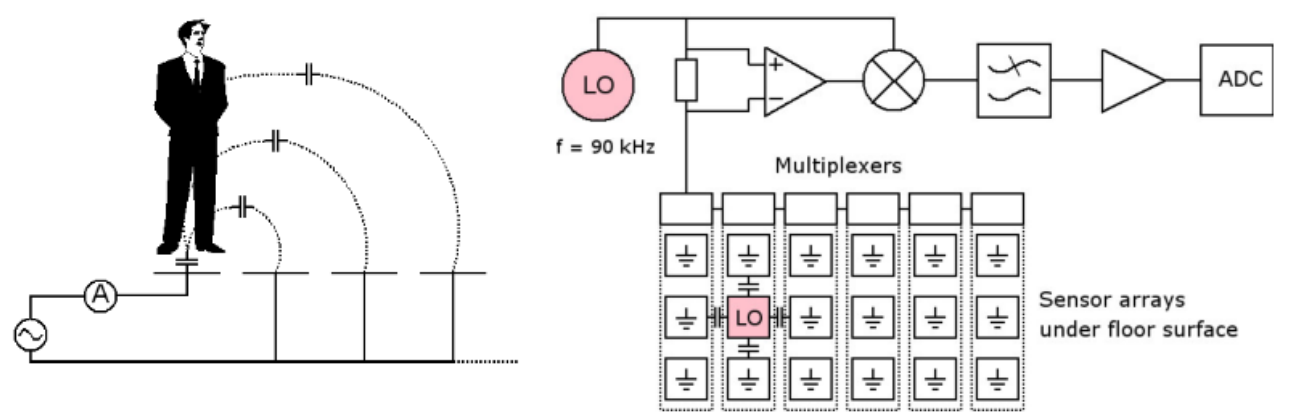

Figure 4. Measurement Principle [14]

In a work conducted by Rimminen et al. [14-16] demonstrated the application of the electric field for human tracking by locating people over a segmented floor electrode. The system was implemented using loading mode. Their design employs a planar electrodes matrix to be placed underneath of the floor in sequence. On aluminum foil, the electrodes are etched and laminated between slim plastic films. As soon as a person comes in standing position over a tile that works as a transmitter. A low frequency signal is transmitted from the transmitted at a time keeping the other tiles grounded. Between the person and the floor, an electric field coupling is produced and thereby influences the current flow as well as impedance. Consequently, the system computes the difference in impedance at a specific frequency. The sensor matrix is then scanned in order by multiplexing the transmitted signal in order to track people efficiently. The authors have determined the person's location's two-dimensional position by making use of the position of the tiles.

In [17] the author envisioned the development of a "smart carpet", a scalable system designed to detect a fall and track people walking over it, with capacitive sensors embedded in the textiles and woven into the carpet. A smart carpet was built on a network of 180 capacitive sensors using one sensor connect to a PC. Each sensor has its own sensing wire placed in a $15 \times 15 \mathrm{~cm}$ size squares with a $5 \mathrm{~cm}$ gap between the sensors. Loading mode is implemented in this device, whereby when a person walks on the carpet, the sensor will be activated, behaving like a transmitter, increasing the current flow to the other tiles which are providing the return path to the ground. Using this carpet, a number of categorization techniques were analysed to identify person's footsteps and thereby assess walking trajectories. The aim of the system was to detect issues such as whether a person is walking on the carpet, or the person is lying flat on the floor. Advantages of this system are that the shape and the size of the carpet can be modified by cutting it between the sensors. Furthermore, the smart carpet does not require the user to remember to maintain the batteries, as it uses "energy scavenging sensors" that harvest energy (i.e. light, thermoelectric and vibrational energy) from the environment. Moreover, [17] revealed that participants found no significant dissimilarities between walking on a standard carpet and walking on the smart carpet and, proposing that this feature would increase the suitability of the fall detector. However, they failed to discuss the robustness of the sensors and the hardware design of the smart carpet is slightly complex. 
CapFloor [18] presented, a system which can localise and detect falls, of a human body using low-intensity electric fields. This system provides a solution for devices which are integrated underneath the floor surface.

CapFloor system is designed with a simple grid network of wires as illustrated in Figure5. The sensors are attached near the walls, which makes them prone to faults, thus there is a simple grid of wires with sensors attached to make the system more faulttolerant, and it can easily be fixed effortlessly if an error occurs.
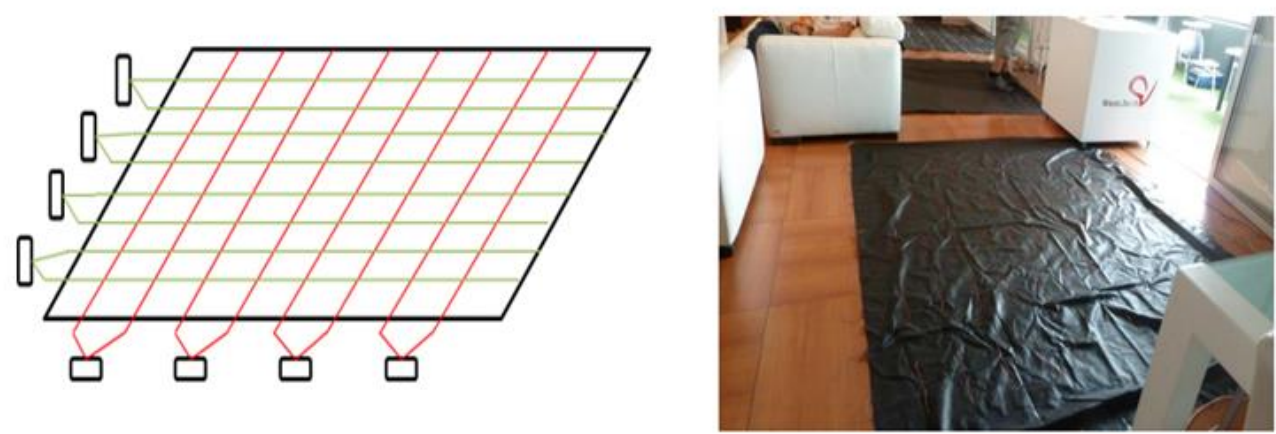

Figure 5. CapFloor Setup [18]

\section{Conclusion}

The review paper aims to identify and recognize the finest practices in the applications of Human-Computer Interaction (HCI). To accomplish this, firstly, an overview over the field of electric field sensing is provided for HCI followed by categorizing the mode of sensing which has been used in the development of the HCI applications. The focus was set to obtain a comprehensive review which included giving reasons why the growth in this field is desirable or even necessary. It showed that electric field sensing can offer a simple and also natural means of control while being very cheap and efficient, the accomplished results are very promising and motivating for future research.

\section{References}

[1] T.G. Zimmerman, J.R. Smith, J.A. Paradiso, D. Allport, and N. Gershenfeld. Applying Electric Field Sensing to Human-Computer Interfaces. Proceedings of the SIGCHI conference on Human factors in computing systems, (1995). Colorado, USA; pp. 280-287.

[2] J.R. Smith. Electric Field Sensing for Graphical Interfaces. IEEE, Computer Graphics and Applications, (1998), 18(3), pp. 54-60.

[3] J.R.. Smith. Electric Field Imaging. (1999). Retrieved 15 September 2014, from http://web.media.mit.edu/ jrs/phd.pdf

[4] T.H. Bullock. Electroreception, (2005), pp. 21.

[5] B. Kramer. Electroreception and Communication in Fishes. Progress in Zoology, (1996), 42.

[6] Gamer, L. (1967). For That Different Sound, Music a'la Theremin. Popular Electronics.

[7] N. Karlsson and J.O. Jarrhed. A capacitive sensor for the detection of humans in a robot cell. IEEE Instrumentation and Measurement Technology Conference, (1993), pp. 164- 166.

[8] P. Dietz and D. Leigh. Diamondtouch: A Multi-User Touch Technology. In Proceedings of the 14th annual ACM symposium on User interface software and technology, (2001), pp. 219-226.

[9] M. Youssef, M. Mah, and A. Agrawala. A Capacitive Sensing Toolkit for Pervasive Activity Detection and Recognition. IEEE International Conference on Pervasive Computing and Communications, (2007), pp. 171- 180.

[10] R. Wimmer, A. Schmidt, P. Holleis, and M. R. Kranz. Thracker Using Capacitive Sensing for Gesture Recognition. Proceedings of the 26th IEEE International Conference on Distributed Computing Systems Workshops (ICDCSW'06). (2006).

[11] M. Valtonen. Technologies for Smart Environments: Capacitive User Tracking and Proactive Fuzzy Control. Ph.D. Thesis, Tampere University of Technology, Department of Electronics. (2012). 
[12] M. Valtonen and J. Vanhala. Human Tracking Using Electric Fields. IEEE International Conference on Pervasive Computing and Communications. (2009), pp. 1-3.

[13] M. Valtonen, L. Kaila, J. Mäentausta and J. Vanhala. Unobtrusive Human Height and Posture Recognition with a Capacitive Sensor. Journal of Ambient Intelligence and Smart Environments. (2011), vol. 3, no. 4, pp. 305-332.

[14] H. Rimminen, M. Linnavuo, and R. Sepponen. Human Tracking using Near Field Imaging. Second International Conference on Pervasive Computing Technologies for Healthcare. (2008), pp. 148-151.

[15] H. Rimminen, M. Linnavuo, and R. Sepponen. Positioning Accuracy and Multi-Target Separation with a Human Tracking System using Near Field Imaging. International Journal on Smart Sensing and Intelligent Systems. (2009), vol. 2, no. 1, pp. 156-175.

[16] H. Rimminen, J. Lindström, M. Linnavuo, and R. Sepponen. Detection of Falls Among the Elderly by a Floor Sensor using the Electric Near Field, (2010), vol. 14, no. (6), pp. 1475-1476.

[17] Aud, M. A., Abbott, C. C., Tyrer, H. W., Neelgund, R. V., Shriniwar, U. G., Mohammed, A., \& Devarakonda, K. K. (2010). Smart Carpet:Developing a Sensor System to Detect Falls and Summon Assistance Myra. Journal of Gerontological Nursing, 36(7), 8-12.

[18] Braun, A., Heggen, H., \& Wichert, R. (2012). CapFloor - A flexible capacitive indoor localization system. Communications in Computer and Information Science, 309 CCIS, 26-35 
International Journal of Smart Home

Vol. 11, No. 4 (2017) 